\title{
Marginal integrity of restorations produced with a model composite based on polyhedral oligomeric silsesquioxane (POSS)
}

\author{
Luciano Ribeiro CORREA NETTO', Alexandre Luiz Souto BORGES ${ }^{2}$, Heloisa Bailly GUIMARÃES ${ }^{1}$, Elis Regina Nunes \\ de ALMEIDA', Laiza Tatiana POSKUS ${ }^{1}$, Eduardo Moreira da SILVA ${ }^{1}$
}

\author{
1- Universidade Federal Fluminense, Faculdade de Odontologia, Laboratório Analítico de Biomateriais Restauradores - LABion-R, Niterói, RJ, Brazil \\ 2- Universidade Estadual Paulista, Departamento de Materiais Odontológicos e Prótese, São José dos Campos, SP, Brazil
}

Corresponding address: Eduardo Moreira da Silva - Faculdade de Odontologia - Universidade Federal Fluminense - Rua Mário Santos Braga, $\mathrm{n}^{\circ} 30$ - Campus Valonguinho - Centro - Niterói - RJ - Brazil - 24020-140 - Phone: 5521 2629-9832 - Fax: 5521 2622-5739 - e-mail: emsilva@vm.uff.br

Submitted: February 20, 2015 - Modification: June 23, 2015 - Accepted: July 28, 2015

\section{ABSTRACT}

\begin{abstract}
$\mathrm{M}$ arginal integrity is one of the most crucial aspects involved in the clinical longevity of resin composite restorations. Objective: To analyze the marginal integrity of restorations produced with a model composite based on polyhedral oligomeric silsesquioxane (POSS). Material and Methods: A base composite (B) was produced with an organic matrix with UDMA/TEGDMA and $70 \mathrm{wt} \%$ of barium borosilicate glass particles. To produce the model composite, 25 wt. \% of UDMA were replaced by POSS (P25). The composites P90 and TPH3 (TP3) were used as positive and negative controls, respectively. Marginal integrity (\%MI) was analyzed in bonded class I cavities. The volumetric polymerization shrinkage $(\% \mathrm{VS})$ and the polymerization shrinkage stress (Pss - MPa) were also evaluated. Results: The values for \%MI were as follows: P90 $(100 \%)=$ TP3 $(98.3 \%)=\mathrm{B}(96.9 \%)>$ P25 $(93.2 \%),(p<0.05)$. The \%VS ranged from $1.4 \%(P 90)$ to $4.9 \%(P 25)$, while Pss ranged from 2.3 MPa (P90) to 3.9 MPa (B). For both properties, the composite P25 presented the worst results $(4.9 \%$ and $3.6 \mathrm{MPa})$. Linear regression analysis showed a strong positive correlation between \%VS and Pss ( $r=0.97)$, whereas the correlation between Pss and \%MI was found to be moderate $(r=0.76)$. Conclusions: The addition of 25 wt. $\%$ of POSS in methacrylate organic matrix did not improve the marginal integrity of class I restorations. Filtek P90 showed lower polymerization shrinkage and shrinkage stress when compared to the experimental and commercial methacrylate composite.
\end{abstract}

Keyw ords: Composite resins. Dental marginal adaptation. Dental stress analysis.

\section{NTRODUCTION}

Nowadays, resin composites are widely used to rebuild dental hard tissues lost by trauma or caries $^{9}$. This class of restorative materials comprises an organic matrix based on methacrylate monomers (Bis-GMA, Bis-EMA, TEGDMA and UDMA), inorganic filler particles and a silane-coupling agent that improves the chemical interaction between the matrix and the fillers ${ }^{9}$. The polymerization of these materials is mediated by photosensitizer substances that absorb photons, reach an excited stage and break the terminal $\mathrm{C}=\mathrm{C}$ bonds of the methacrylate monomers producing a free radical copolymerization reaction ${ }^{23}$.
Irrespective of the improved mechanical and aesthetic properties, however, volumetric shrinkage still remains a shortcoming to be overcome when using resin composites ${ }^{6}$. The rapid reduction in volume that occurs when the composite changes from a viscous to a rigid state may develop stresses at the tooth-restoration interface, leading to loss of marginal integrity and postoperative sensitivity ${ }^{16}$.

Clinically, techniques such as C-factor control, incremental filling and modulation of the irradiance used for photocuring the composite are used in an attempt to minimize the effects of polymerization shrinkage stresses 8,31 . In the field of materials science, some studies have focused on changes in the material composition such as inorganic filler 
type and loading ${ }^{25}$, the use of high molecular weight monomers ${ }^{24}$ and especially the use of monomers with a chemical structure that promotes low polymerization shrinkage ${ }^{22}$. In the last decade, a new organic matrix based on silorane monomers was introduced ${ }^{19}$. The silorane molecule comprises a hydrophobic siloxane core with oxiranic terminations that initiates a cationic ring-opening polymerization that counteracts the loss of volume due to polymeric network formation ${ }^{29}$. Theoretically, this mechanism leads to lower polymerization shrinkage. In fact, published studies have shown that resin composites based on silorane presented reduced gap formation at the tooth-composite interface ${ }^{16}$. Due to a complex photocuring system mediated by a cationic initiator and an acid salt of iodine, these composites are chemically incompatible with composites based on methacrylate monomers ${ }^{11}$. This aspect could be seen as a clinical limitation of the silorane-based materials.

Recent studies have shown promising results regarding the volumetric shrinkage in methacrylate organic matrixes modified with polyhedral oligomeric silsesquioxane (POSS) ${ }^{10,27,30}$. POSS is a nanostructured hybrid (organic-inorganic) molecule with an empirical formula $\mathrm{RSiO}_{1,5}$, where $\mathrm{SiO}_{1,5}$ corresponds to a core of $\mathrm{SiO}$ and $\mathrm{R}$ can be a hydrogen atom or any functional pendant group on its eight vertices, for example, a functional methacrylate group ${ }^{17}$. As a result, the POSS molecule can be copolymerized with a dimethacrylate system using a conventional camphorquinone/tertiary amine photoinitiation system ${ }^{30}$.

Former studies have tested ternary organic matrixes of Bis-GMA/TEGDMA/POSS, with positive results only when a low content of POSS (2-10 wt. \%) was used ${ }^{10,30}$. These results were probably influenced by the high viscosity of Bis-GMA, which can impair the mobility of the organic matrixes ${ }^{23}$. Taking this into consideration, an experimental resin composite with a ternary UDMA/TEGDMA/ POSS organic matrix was formulated in the present study. The UDMA is an aliphatic molecule without pendant $-\mathrm{OH}$ groups, a feature that causes this monomer to be less viscous than Bis-GMA ${ }^{23}$. Theoretically, these characteristics could allow a higher incorporation of POSS with better results in terms of polymerization shrinkage. Therefore, the purpose of the present study was to analyze the marginal integrity of restorations produced with a model composite based on polyhedral oligomeric silsesquioxane (POSS). The research hypothesis was that the model composite based on POSS would present low volumetric polymerization shrinkage, reduced shrinkage stresses and better marginal integrity than the other tested composites.

\section{MATERIAL AND METHODS}

The materials used in the present study are depicted in Figure 1. The monomers UDMA, TEGDMA (Essthec, Inc. Essington, PA, USA) and POSS (Hybrid plastics, Inc., Fountain Valley, CA, USA) were used as received. The POSS molecule used was methacrylPOSS, which has organic methacrylate functional groups attached at the eight corners of the SiO cage. $0.6 \mathrm{wt}$ \% of camphorquinone and $1.2 \mathrm{wt} \%$ of ethyl N, N-dimethyl-4-aminobenzoate (EDMAB, Aldrich Chemical Company, Inc., Milwaukee, WI, USA) were incorporated as photosensitizer and reducing agents. The inorganic phase of the materials was composed of $70 \mathrm{wt}$ \% of silanized barium borosilicate particles with an average size of $0.7 \mu \mathrm{m}$ (Essthec, Inc. Essington, PA, USA). All the components (monomers, filler particles and photosensitizers) were weighed using an analytical balance (AUW 220D, Shimadzu, Tokyo, Japan) and mixed in a dual centrifuge (150.1 FVZ SpeedMixer DAC, FlackTek Inc., Herrliberg, Germany). The monomers were mixed to make neat UDMATEGDMA and UDMA-TEGDMA-POSS matrixes. Then, the photosensitizer and the reducing agent were added to the neat matrixes and centrifuged at $1300 \mathrm{rpm}$ for $1 \mathrm{~min}$. Finally, the filler particles were incorporated and the mixture was homogenized at $2400 \mathrm{rpm}$ for $2 \mathrm{~min}$.

The resin composites P90, a commercially available low shrinkage material based on silorane molecule, and TPH3, a traditional methacrylate composite, were used as positive and negative controls, respectively.

All the specimens used in the present study were photoactivated with a quartz-tungsten-halogen unit (Optilux 501, Demetron Danburry Inc., USA), using an irradiance of $650 \mathrm{~mW} / \mathrm{cm}^{2}$ for $40 \mathrm{~s}$ (radiant exposure of $26 \mathrm{~J} / \mathrm{cm}^{2}$ ).

\section{Volumetric polymerization shrinkage ( $\%$ VS)}

The \%VS was measured using a video-imaging device (AcuVol, Bisco Inc., Schaumburg, IL, USA) ${ }^{15}$. Five specimens of each uncured composite, with a volume of $10 \mu \mathrm{L}$, were manually manipulated into a semispherical shape and placed on a teflon (polytetrafluoroethylene) rotational pedestal inside the AcuVol chamber and in front of the CCD camera from the equipment. After $5 \mathrm{~min}$, the specimens were photoactivated with the tip of the light-curing unit positioned $1 \mathrm{~mm}$ above the specimen. The \%VS was measured 5 min after photoactivation. The data were processed by the AcuVol software.

Polymerization shrinkage stress (Pss)

The Pss was measured using a Pss device (ODMT03d, Odeme Biothecnology, Joaçaba, 
SC, Brazil) fixed to a universal testing machine equipped with a load cell of 50 N (EMIC DL-2000; São José dos Pinhais, PR, Brazil). Two polymethyl methacrylate rods $(2.0 \mathrm{~mm}$ in diameter and 20 $\mathrm{mm}$ in length, high compliance system) had one of their flat surfaces ground with \#180 SiC paper, treated with methyl methacrylate (Jet, Clássico Artigos Odontológicos, São Paulo, SP, Brazil) for 10 $S$ and coated with the specific adhesive system for each composite (Table 1 ). The rods were attached to the lower and upper clamps of the Pss device, maintaining a distance of $2 \mathrm{~mm}$ between the flat

\begin{tabular}{|c|c|c|c|}
\hline Material & Composition & Adhesive system & Mode of application \\
\hline TPH3 & $\begin{array}{c}\text { Matrix: Urethane modified } \\
\text { Bis-GMA; Bis-EMA; } \\
\text { TEGDMA }\end{array}$ & Clearfil SE Bond & $\begin{array}{l}\text { Primer was applied on the cavity } \\
\text { surface for } 20 \mathrm{~s} \text { and then gently } \\
\text { air dried. The bond was applied, } \\
\text { gently air dried and photoactivated } \\
\text { for } 10 \mathrm{~s} \text {. }\end{array}$ \\
\hline (TP3) & $\begin{array}{l}\text { Filler (75 wt.\%): BaAIBSi; } \\
\text { BaFAIBSi; highly dispersed } \\
\text { silicon dioxide }(0.02-1 \mu \mathrm{m})\end{array}$ & & \\
\hline Filtek P90 & $\begin{array}{c}\text { Matrix: 3, 4-epoxy } \\
\text { cyclohexyl cyclopolymethyl } \\
\text { siloxane }\end{array}$ & $\begin{array}{l}\text { Self-etching bond P90 - } \\
\text { AP90 }\end{array}$ & $\begin{array}{l}\text { Primer was applied for } 10 \mathrm{~s} \text {, gently } \\
\text { air dried, and light cured for } 10 \mathrm{~s} . \\
\text { Bond was applied, air thinned, and } \\
\text { photoactivated for } 10 \mathrm{~s} .\end{array}$ \\
\hline (P90) & $\begin{array}{c}\text { Filler (76 wt. \%): silanized } \\
\text { quartz; Yttrium fluoride }(0.05 \\
-5 \mu \mathrm{m})\end{array}$ & & \\
\hline \multirow[t]{2}{*}{$B$} & $\begin{array}{c}\text { Matrix: } 70 \text { wt. } \% \text { UDMA, } 30 \\
\text { wt. } \% \text { TEGDMA }\end{array}$ & Clearfil SE Bond & $\begin{array}{l}\text { Primer was applied on the cavity } \\
\text { surface for } 20 \mathrm{~s} \text { and then gently } \\
\text { air dried. The bond was applied, } \\
\text { gently air dried and photoactivated } \\
\text { for } 10 \mathrm{~s} \text {. }\end{array}$ \\
\hline & $\begin{array}{c}\text { Filler: } 70 \text { wt. } \% \text { of silanized } \\
\text { barium borosilicate particles } \\
\text { with an average size of } 0.7 \\
\mu \mathrm{m}\end{array}$ & & \\
\hline \multirow[t]{2}{*}{ P25 } & $\begin{array}{c}\text { Matrix: } 45 \text { wt. } \% \text { UDMA, } 30 \\
\text { wt. } \% \text { TEGDMA, } 25 \text { wt. } \% \\
\text { POSS }\end{array}$ & Clearfil SE Bond & $\begin{array}{l}\text { Primer was applied on the cavity } \\
\text { surface for } 20 \mathrm{~s} \text { and then gently } \\
\text { air dried. The bond was applied, } \\
\text { gently air dried and photoactivated } \\
\text { for } 10 \mathrm{~s} .\end{array}$ \\
\hline & $\begin{array}{c}\text { Filler: } 70 \text { wt. } \% \text { of silanized } \\
\text { barium borosilicate particles } \\
\text { with an average size of } 0.7 \\
\mu \mathrm{m}\end{array}$ & & \\
\hline
\end{tabular}

Figure 1- Materials used in the present study

Table 1- Means \pm standard deviations of $\%$ VS, Pss and \%Ml

\begin{tabular}{|c|c|c|c|}
\hline Composite & $\%$ VS & Pss - MPa & $\%$ MI \\
\hline $\mathrm{B}$ & $4.8^{\mathrm{C}} \pm 0.2$ & $3.9^{\mathrm{B}} \pm 0.3$ & $96.9^{\mathrm{A}, \mathrm{B}} \pm 0.4$ \\
\hline P25 & $4.9^{c} \pm 0.2$ & $3.6^{\mathrm{B}} \pm 0.4$ & $93.2^{\mathrm{B}} \pm 0.4$ \\
\hline TP3 & $3.2^{\mathrm{B}} \pm 0.1$ & $2.8^{\mathrm{A}} \pm 0.4$ & $98.3^{A} \pm 0.3$ \\
\hline P90 & $1.4^{\mathrm{A}} \pm 0.1$ & $2.3^{A} \pm 0.4$ & $100^{A} \pm 0.0$ \\
\hline
\end{tabular}

In each column, values with identical letters are similar (Tukey HSD, $p>0.05$ ) 
treated surfaces ${ }^{2}$. The composite was inserted between the flat treated surfaces, in the shape of a cylinder, following the perimeter of the rods (C-Factor: 0.5 ) and photoactivated at a distance of $2 \mathrm{~mm}$ from its lateral. Specimen height was kept constant by the feedback from an extensometer (2630-101, Instron, Norwood, MA, USA) attached to the polymethyl methacrylate rods. The composite contraction force was monitored for 10 min after photoactivation, and the Pss ( $\mathrm{MPa}$ ) was obtained by dividing the maximum force value $(\mathrm{N})$ by 3.14 $\left(\pi r^{2}\right),(n=5)$.

\section{Marginal integrity ( \% MI)}

Ten human molars free of caries, defects and cracks were cleaned and immersed in a $1 \%$ aqueous solution of chloramine for seven days, rinsed and frozen in distilled water until use. The roots were removed and the crowns were sectioned in a buccal-lingual direction to produce two halves. The mesial and distal surfaces of the teeth were flattened using \#600 SiC paper (DPU 10, Struers, Denmark) until enamel surfaces with an area of $4.0 \mathrm{~mm} \times 4.0 \mathrm{~mm}$ were produced (MPI/E-101, Mitutoyo, Tokyo, Japan). One class I cavity ( $\varnothing$ $=4.0 \mathrm{~mm}$ and $\mathrm{h}=2.0 \mathrm{~mm}$ ) was prepared on each flat enamel surface using a cylindrical diamond bur (\#3053, KG Sorensen, SP, Brazil) attached to a standardized cavities device (LABiom-R, UFF, Niterói, RJ, Brazil) under copious water-cooling. The tooth halves with cavities were randomly divided into four groups $(n=5)$ according to the composite used for the restorations.

The cavities were washed with distilled water, dried with tissue paper and hybridized with the proper adhesive system, following the manufacturers' instructions (Figure 1). After applying the respective adhesive system, the cavities were bulk restored and the composite was photoactivated from the occlusal surface. The specimens were then stored for seven days in distilled water at $37^{\circ} \mathrm{C}$, immersed in $1 \%$ methylene blue solution for $2 \mathrm{~h}$, rinsed with distilled water and air-dried.

The analysis of \%MI was carried out using a stereomicroscope (Olympus SZ61, Olympus Corporation, Tokyo, Japan) equipped with a digital camera (Color view, Olympus Corporation, Tokyo, Japan). First, images of each restorative surface were captured at X40 magnification. After that, the digitalized images were analyzed using the Image J software (National Institute of Mental Health, Bethesda, USA $)^{1,28}$. The \%MI was calculated as the ratio of the stained cavity interface to the entire cavity perimeter by using the following formula:

$$
\% M I=\frac{M I}{2 p r} \times 100 \text {, }
$$

where MI is the margins without gaps and $r$ is the radius of the cavity.

In order to confirm the \%MI obtained using stereomicroscopy, specific areas of the specimens, where gaps were more stained by the methylene blue solution, were analyzed under scanning electron microscope (SEM). The specimens were mounted in a charge reduction sample holder and observed under SEM (PhenomProX, PhenomWorld, Eindhoven, The Netherlands) operating in the backscattered mode, in a low vacuum environment. The SEM images were taken at a magnification of X2500.

Additionally, the specimens were embedded in epoxy resin and sectioned through the center of the restorations (Isomet 1000 Precision Saw, Buehler, Lake Bluff, IL, USA). The surfaces obtained were wet-polished with \#600, 1200 and 4000 SiC papers, ultrasonicated for $5 \mathrm{~min}$ and submitted to internal

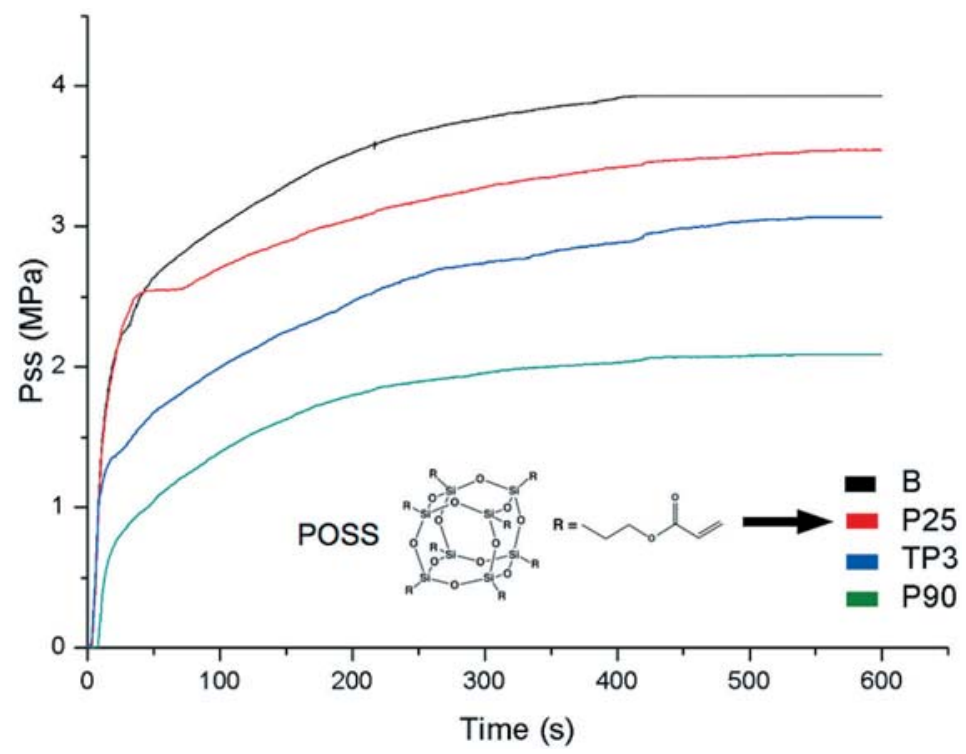

Figure 2- Plot of Pss (MPa) vs. time $(0-600 \mathrm{~s})$ for the materials tested 
gap evaluation at X40 magnification (Olympus SZ61, Olympus Corporation, Tokyo, Japan).

\section{Statistical analysis}

The data obtained were analyzed using Statgraphics Centurion XVI software (STATPOINT Technologies, Inc, USA). Initially, the normal distribution of errors and the homogeneity of variances were checked by the Shapiro-Wilk's test and Levene's test. Based on these preliminary analyses, the data for each variable (\%VS, SS and $\% \mathrm{MI}$ ) were individually analyzed using one-way ANOVA and Tukey's HSD post hoc test. The data were also submitted to linear regression analysis: Pss vs. \%VS and \%MI vs Pss. All analyses were performed at a significance level of $\alpha=0.05$.
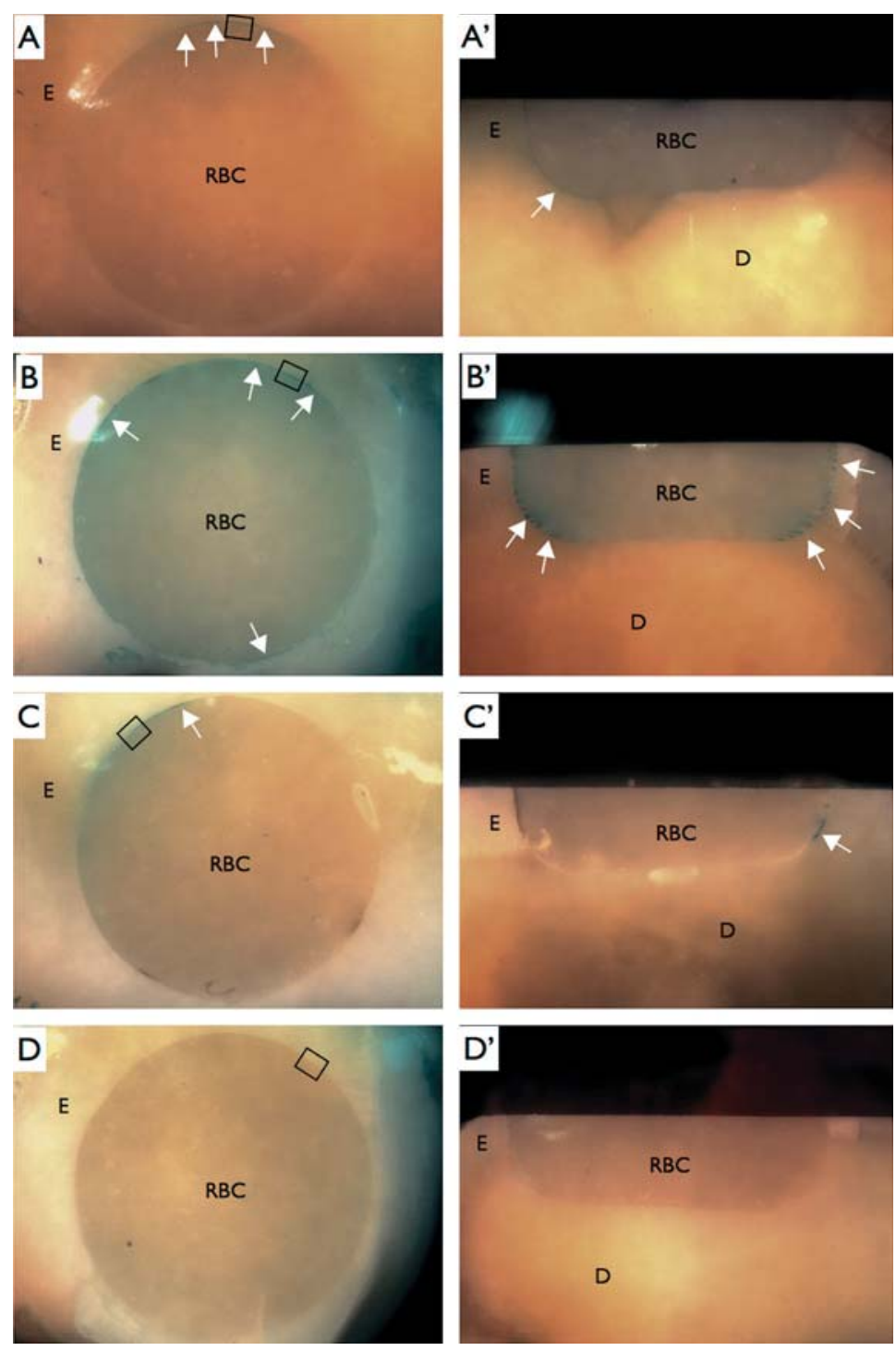

Figure 3- Representative optical microscopy images of cavities restored with the four resin composite: B (AA'); P25 (BB'); TP3 (CC') and P90 (DD'). RBC = resin-based composite, E = enamel, D = dentin. White arrows show gaps marked with methylene blue at the cavity wall-RBC interfaces. Black rectangles are areas selected for scanning electron microscope analysis

\section{RESULTS}

Table 1 summarizes the results of \%VS, Pss and \%MI. P90 presented the lowest \%VS, followed by TP3, whereas the experimental composites B and P25 presented similar and higher \%VS $(p<0.05)$.

Regarding Pss, P90 and TP3 presented similar and lower values than B and P25, which were not statistically different from each other $(p>0.05)$. Figure 2 shows representative plots of Pss (MPa) vs. time (0 - $600 \mathrm{~s})$ for each composite tested. The differences in behavior among the materials were remarkable.

With respect to \%MI, the highest values were presented by P90, TP3 and B, without significant differences among them ( $p>0.05)$. The lowest \%MI was presented by $\mathrm{P} 25$, although its value was not statistically different from that of B. Figure 3 shows 

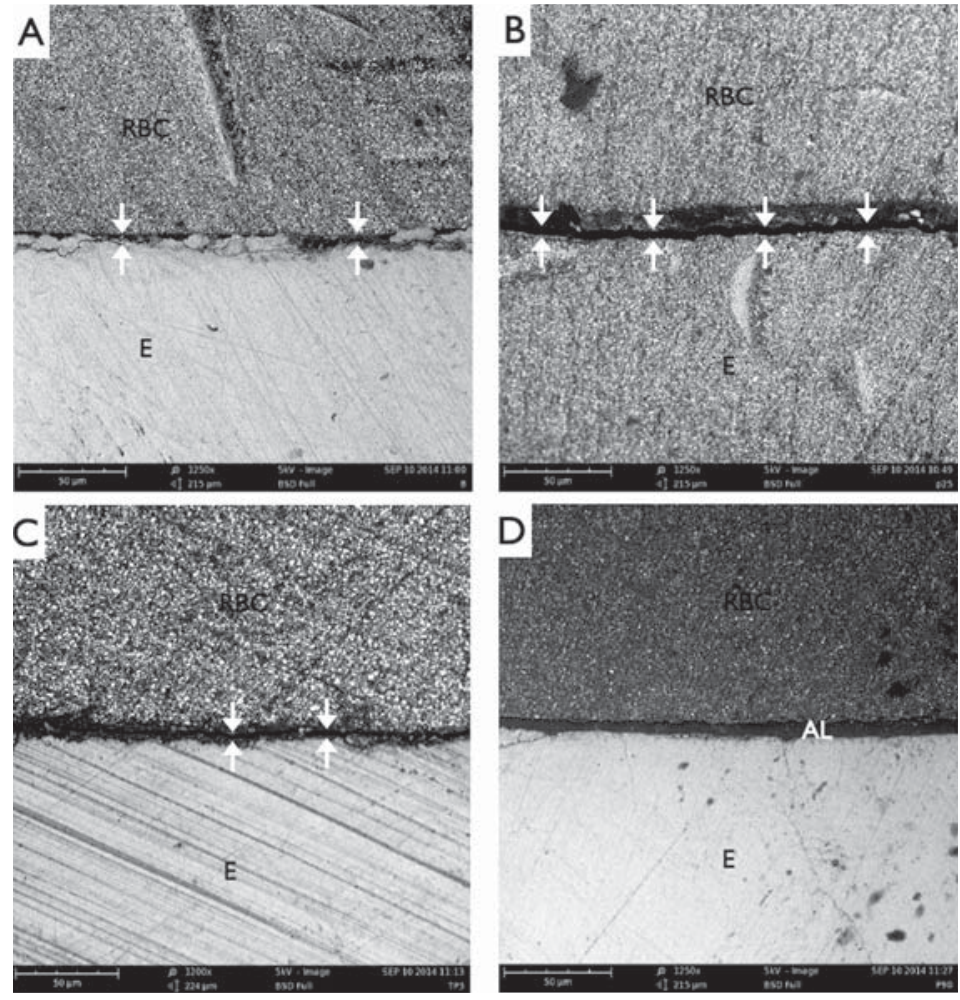

Figure 4- Scanning electron microscope micrographs of the areas indicated by the black rectangles in Figure 3 . RBC = resin-based composite, $\mathrm{E}=$ enamel, $\mathrm{AL}=$ adhesive layer. White arrows show gaps at the cavity wall-RBC interfaces

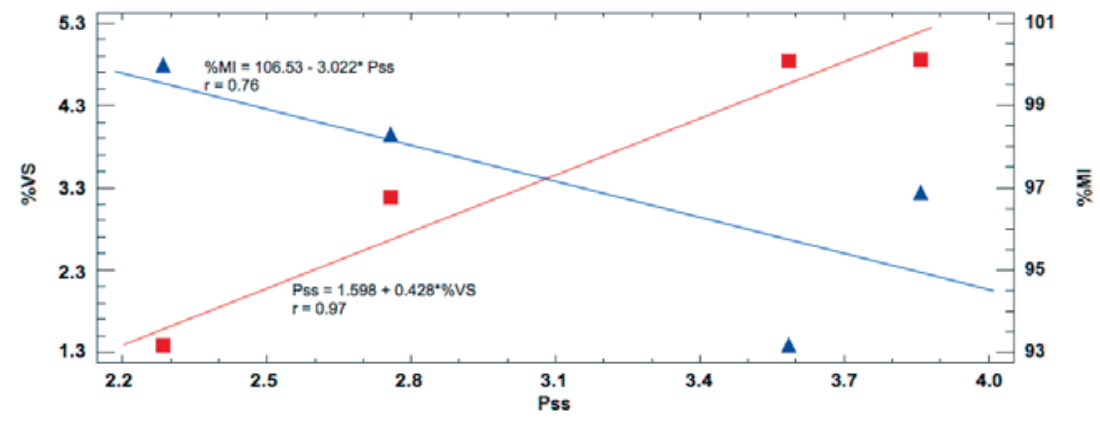

Figure 5- Linear regression plots of Pss vs. \%VS and \%MI vs Pss

representative optical microscope images of cavities restored with the four resin composites. Figure 4 shows SEM images of the areas pointed out by the White arrows in Figure 3.

The linear regression analysis showed a strong positive correlation between Pss and \%VS ( $r=0.97)$, whereas the correlation between \%MI and Pss was found to be negative and moderate $(r=-0.76)$ (Figure 5).

\section{DISCUSSION}

The results obtained by Fong, et al. ${ }^{10}$ (2005) and by $\mathrm{Wu}$, et al. ${ }^{30}$ (2010) were one of the motivators in developing the present study. Those authors found that the replacement of Bis-GMA by POSS did not affect or decrease the polymerization shrinkage of experimental composites with binary Bis-GMA/
TEGDMA (50/50 wt.\%) organic matrixes. The first study used matrixes modified with $2 ; 10 ; 25$ and 50 wt.\% of POSS, while the second employed replacements of $2 ; 5 ; 10$; and 15 wt.\%. The second motivator was based on the own features of the inorganic phase of POSS molecule $\left(\mathrm{SiO}_{1,5}\right)$, a silsesquioxane cage that can act as a nanofiller. In this field, it is well established that the greater the filler particle content, the lower the polymerization shrinkage of the resin composites ${ }^{26}$, and regular filler particles, such as POSS, may also contribute to this reduction ${ }^{25}$. Another important aspect is that these POSS nanofillers are not silanized and could induce less internal stress during the polymerization of the composite ${ }^{4}$.

In the study of $\mathrm{Wu}$, et al. ${ }^{30}$ (2010), the increase in the POSS content from 0 to 15 wt. \% reduced the polymerization shrinkage from $3.53 \%$ to $2.18 \%$. According to these authors, this occurred because 
the change in free volume inside the crosslinked polymer network was limited by the nanocubic structures of POSS. Based on this theory, it is reasonable to infer that the increase in the content of POSS could further reduce the shrinkage develop by the composite. This was the rationale for using UDMA instead of Bis-GMA in the current study. Due to the low viscosity of UDMA, it was possible to produce an experimental composite with only 30 wt. $\%$ of TEGDMA while adding 25 wt. $\%$ of POSS. This low content of TEGDMA can also contribute to the reduction in polymerization shrinkage and polymerization stress of resin composites ${ }^{12}$.

The evaluation of an experimental composite with 25 wt.\% of POSS was based on results obtained in our Lab (unpublished data), which showed that among experimental composites with $2 ; 5 ; 10 ; 25$ and 50 wt.\% of POSS, the material with 25 wt. \% presented the best overall results in terms of physical-mechanical properties (degree of conversion, hardness, flexural strength, young's modulus, water sorption and solubility).

After photoactivation, a chain of phenomena will take place inside the composite. Firstly, the 0.3-0.4 $\mathrm{nm}$ intermolecular spaces between the dimethacrylate monomers of the organic matrix, maintained by Van der Waals forces, are reduced by the conversion of the $\mathrm{C}=\mathrm{C}$ bonds and the establishment of $\mathrm{C}-\mathrm{C}$ bonds with lengths of 0.15 $\mathrm{nm}$ between polymer chains ${ }^{23,30}$. The result is a volumetric polymerization shrinkage ranging from 2 to $5 \%{ }^{18}$. This polymerization kinetic involves three phases: the pre-gel phase, gel point and post-gel phase. The pseudoplastic behavior of the organic matrix in the pre-gel phase is able to release the stresses derived from shrinkage. However, when the matrix reaches the gel point and suffers a transformation from a viscous to a rigid state, the relaxation of shrinkage drastically drops. Finally, in the post-gel phase, the mass of composite is fully rigid and the stresses are trapped inside the material. In a cavity restored with a composite material, these phenomena are restricted by the adhesion provided by the adhesive system previously applied on the cavity walls, and the stresses are transferred to the tooth wall-composite interface. Thus, if the bonded strength is lower than the stresses generated during the polymerization, gap formation at the tooth-composite interface will take place ${ }^{21}$.

P25 presented the highest \%VS and Pss (Table 1 ). These results led to the rejection of the hypothesis of the present study. Based on the findings of $\mathrm{Wu}$, et al. ${ }^{30}$ (2010) these results were somewhat surprising. The own structure of POSS, however, can be used to explain this behavior. In other words, it is reasonable to claim that the eight methacrylate terminations at the corners of the silsesquioxane cage of POSS increased the $\mathrm{C}=\mathrm{C}$ bonds to volume ratio that were broken during P25 photoactivation, and led to a higher packing density in the formed polymeric network ${ }^{7}$. Moreover, these methacrylate groups may have also developed a highly heterogeneous crosslinking network, which could have increased the shrinkage stress inside the material. Furthermore, "non-silanized POSS nanofillers" did not contribute to less internal stress in P25. It is possible that in the post-gel phase, the POSS cages anchored into the crosslinking network and hindered the mobility of the polymeric chains, thereby generating greater internal stress in the polymeric network.

Even without POSS incorporation, B presented $\%$ VS and Pss values statistically similar to P25. This behavior can be related to the presence of 30 wt. \% of TEGDMA in this experimental composite. TEGDMA is a low molecular weight monomer that can negatively affect polymerization shrinkage from two paths. First, the low viscosity of TEGDMA allows this monomer to flow easily, thereby increasing the matrix mobility ${ }^{12}$. Second, the greater amount of $\mathrm{C}=\mathrm{C}$ bonds to volume ratio may increase the degree of conversion and produce a high packing density in the matrix. These concepts agree with previous studies, which indicated that low Bis-GMA: or UDMA:TEGDMA ratios increased the polymerization shrinkage of experimental composites ${ }^{12,13}$.

The \%Vs and Pss are dynamic phenomena strongly influenced by the filler loading 25,26 and the type of monomers present in resin composite's organic matrix ${ }^{22,24}$. TP3 (75 wt.\%) and P90 (76 wt.\%) present similar filler loading (Figure 1 ). Considering this, it is reasonable to infer that their \%VS and Pss were influenced more by their organic matrixes than by filler particles. TP3 presented intermediary $\%$ VS and Pss. Although the exact content of the monomers present in its organic matrix is unknown, we can infer that the higher molecular weight and viscosity of Bis-GMA and Urethane modified BisGMA influenced these results ${ }^{24}$. The best behavior in terms of \%VS was presented by P90 (Table 1), which agrees with previous studies ${ }^{16,19}$. P90 has an organic matrix based on silorane monomer, whose polymerization involves a cationic ring-opening reaction that compensates the shrinkage developed during the formation of the polymeric network ${ }^{15,29}$. On the other hand, the Pss of P90 (2.3 MPa) was not statistically different from that of TP3 (2.8 MPa). Pss is a complex phenomenon ${ }^{3}$, not only dependent on shrinkage but also on the elastic modulus of the material ${ }^{20}$. In other words, the higher the stiffness, the greater the Pss developed during the composite polymerization. Analyzing several low-shrinkage composites, Boaro, et al. ${ }^{2}$ (2010) demonstrated that P90 developed the highest polymerization stress, even presenting the lower volumetric shrinkage, 
and explained this finding based on the high initial flexural modulus of P90. Thus, it can be assumed that the flexural modulus of P90 influenced its Pss in the present study. The significant positive correlation between polymerization stress and flexural modulus presented in the study of ElDamanhoury and Plat ${ }^{5}$ (2014) may reinforce this possibility.

Marginal integrity is one of the most crucial aspects involved in the clinical longevity of resin composite restorations ${ }^{14}$. Thus, in vitro evaluations of this response may contribute to predict the clinical behavior of composite restorative materials ${ }^{13}$. The Class I cavity model used in the present study represents the worst $\mathrm{C}$-factor scenario regarding the development of Pss in resin composite restorations ${ }^{8}$. This cavity model was used in an endeavor to subject the composites to a hard test condition.

P25 presented the worst \%MI (Table 1 ). This aspect is proved in Figure 3, in which the presence of stained gaps is remarkable in the cavity restored with this experimental composite. Moreover, the SEM analysis of the selected areas in each cavity (black rectangles) confirmed this finding (Figure 4). Additionally, B also presented the worst \%MI. In agreement with previous studies ${ }^{13,21}$, the strong positive correlation found between \%VS, and Pss and the negative correlation observed between Pss and \%MI (Figure 5) reinforce the explanation that these phenomena are connected and could explain these findings regarding \%MI.

Most of the internal gaps in the current study were observed at the axiopulpal angles (Figure $3 A^{\prime}$; $B^{\prime}$ and $C^{\prime}$ ). This finding agrees with those of SouzaJunior, et al. ${ }^{28}$ (2011) and clearly demonstrates the competition between bond strength and Pss in this area. P90 was the only material that presented $100 \%$ of marginal integrity (Table 1 ). This finding can be explained by the lowest values of \%VS and Pss presented by this composite.

Although the experimental composite with POSS did not improve the marginal integrity in the present study, future studies should be conducted to analyze the influence of other types of POSS molecules (epoxides, for example) concerning this response.

\section{CONCLUSIONS}

Within the limitations of this investigation, the addition of 25 wt. $\%$ of POSS in a methacrylate organic matrix did not reduce the volumetric polymerization shrinkage and the shrinkage stress, and it did not improve the marginal integrity of restorations. Filtek P90 showed the lowest polymerization shrinkage.

\section{ACKNOWLEDGEMENTS}

This study was supported by CNPq - National Council for Scientific and Technological Development (Grant no. 484094/2012-2). The authors thank ESSTHEC Inc., Essington, PA, USA for donating all the methacrylate monomers and glass fillers.

\section{REFERENCES}

1- Alonso RC, Correr GM, Cunha LG, Borges FS, Puppin-Rontani RM, Sinhoreti MA. Dye staining gap test: an alternative method for assessing marginal gap formation in composite restorations. Acta Odontol Scand. 2006;64:141-5.

2- Boaro LC, Gonçalves F, Guimarães TC, Ferracane JL, Versluis A, Braga RR. Polymerization stress, shrinkage and elastic modulus of current low-shrinkage restorative composites. Dent Mater. 2010;26:1144-50.

3- Borges AL, Borges $A B$, Xavier TA, Bottino MC, Platt JA. Impact of quantity of resin, $\mathrm{C}$-factor, and geometry on resin composite polymerization shrinkage stress in Class $\mathrm{V}$ restorations. Oper Dent. 2014;39:144-51.

4- Condon JR, Ferracane JL. Reduced polymerization stress through non-bonded nanofiller particles. Biomaterials. 2002;23:3807-15. 5- El-Damanhoury HM, Platt JA. Polymerization shrinkage stress kinetics and related properties of bulk-fill resin composites. Oper Dent. 2014;39:374-82.

6- El-Shamy H, Saber MH, Dorfer CE, El-Badrawy W, Loomans $B A$. Influence of volumetric shrinkage and curing light intensity on proximal contact tightness of class II resin composite restorations: in vitro study. Oper Dent. 2012;37:205-10.

7- Fadaie P, Atai M, Imani M, Karkhaneh A, Ghasaban S. Cyanoacrylate-POSS nanocomposites: novel adhesives with improved properties for dental applications. Dent Mater. 2013;29:e61-9.

8- Feilzer AJ, De Gee AJ, Davidson CL. Setting stress in composite resin in relation to configuration of the restoration. J Dent Res. 1987;66:1636-9.

9- Ferracane JL. Resin composite - state of the art. Dent Mater. 2011;27:29-38.

10- Fong $\mathrm{H}$, Dickens SH, Flaim GM. Evaluation of dental restorative composites containing polyhedral oligomeric silsesquioxane methacrylate. Dent Mater. 2005;21:520-9.

11- Gao BT, Lin H, Zheng G, Xu YX, Yang JL. Comparison between a silorane-based composite and methacrylate-based composites: shrinkage characteristics, thermal properties, gel point and vitrification point. Dent Mater J. 2012;31:76-85.

12- Gonçalves F, Azevedo CL, Ferracane JL, Braga RR. BisGMA/ TEGDMA ratio and filler content effects on shrinkage stress. Dent Mater. 2011;27:520-6.

13- Gonçalves F, Pfeifer CC, Stansbury JW, Newman SM, Braga RR. Influence of matrix composition on polymerization stress development of experimental composites. Dent Mater. 2010;26:697-703.

14- Heintze SD. Clinical relevance of tests on bond strength, microleakage and marginal adaptation. Dent Mater. 2013;29:5984.

15- Karaman E, Ozgunaltay G. Polymerization shrinkage of different types of composite resins and microleakage with and without liner in class II cavities. Oper Dent. 2014;39:325-31.

16- Kim H, Park S. Measurement of the internal adaptation of resin composites using micro-CT and its correlation with polymerization shrinkage. Oper Dent. 2014;39:e57-70.

17- Kuo SW, Chang FC. POSS related polymer nanocomposites. Prog Polym Sci. 2011;36:1649-96.

18- Lee IB, Cho BH, Son $\mathrm{HH}$, Um CM. A new method to measure the polymerization shrinkage kinetics of light cured composites. J Oral Rehabil. 2005;32:304-14. 
19- Lien W, Vandewalle KS. Physical properties of a new siloranebased restorative system. Dent Mater. 2010;26:337-44.

20- Moreira da Silva E, Santos GO, Guimarães JG, Barcellos AA, Sampaio EM. The influence of C-factor, flexural modulus and viscous flow on gap formation in resin composite restorations. Oper Dent. 2007;32:356-62.

21- Papadogiannis D, Kakaboura A, Palaghias G, Eliades G. Setting characteristics and cavity adaptation of low-shrinking resin composites. Dent Mater. 2009;25:1509-16.

22- Park HY, Kloxin CJ, Fordney MF, Bowman CN. Stress relaxation of trithiocarbonate-dimethacrylate-based dental composites. Dent Mater. 2012;28:888-93.

23- Peutzfeldt A. Resin composites in dentistry: the monomer systems. Eur J Oral Sci. 1997;105:97-116.

24- Rüttermann S, Dluzhevskaya I, Grosssteinbeck C, Raab WH, Janda R. Impact of replacing Bis-GMA and TEGDMA by other commercially available monomers on the properties of resin-based composites. Dent Mater. 2010;26:353-9.

25- Satterthwaite JD, Maisuria A, Vogel K, Watts DC. Effect of resin-composite filler particle size and shape on shrinkage-stress. Dent Mater. 2012;28:609-14.
26- Shah PK, Stansbury JW. Role of filler and functional group conversion in the evolution of properties in polymeric dental restoratives. Dent Mater. 2014;30:586-93.

27- Soh MS, Yap AU, Sellinger A. Physicomechanical evaluation of low-shrinkage dental nanocomposites based on silsesquioxane cores. Eur J Oral Sci. 2007;115:230-8.

28- Souza-Junior EJ, Souza-Régis MR, Alonso RC, Freitas AP, Sinhoreti MA, Cunha LG. Effect of the curing method and composite volume on marginal and internal adaptation of composite restoratives. Oper Dent. 2011;36:231-8.

29- Weinmann W, Thalacker C, Guggenberger R. Siloranes in dental composites. Dent Mater. 2005;21:68-74.

30- Wu X, Sun Y, Xie W, Liu Y, Song X. Development of novel dental nanocomposites reinforced with polyhedral oligomeric silsesquioxane (POSS). Dent Mater. 2010;26:456-62.

31- Wydra JW, Cramer NB, Stansbury JW, Bowman CN. The reciprocity law concerning light dose relationships applied to BisGMA/TEGDMA photopolymers: theoretical analysis and experimental characterization. Dent Mater. 2014;30:605-12. 